\title{
La enseñanza de las tecnologías de la información geográfica (TIG) en España y en Europa
}

Bosque Sendra, J., Gómez Delgado, M., Aguilera Benavente, F. , Rodríguez Espinosa, V.M., Barreira González, P. y Salado García, M.J.

\section{Resumen:}

En este trabajo se ha realizado una revisión de los estudios TIG que, en la actualidad, se imparten en Grados y Másteres de Universidades de España y Europa. A través de la información recogida en sus planes de estudio, disponible en Internet, se han analizado sus principales características, objetivos, duración, contenidos, asignaturas, etc. El objetivo final del trabajo ha sido formular una primera clasificación de estudios existentes, conocer cómo se insertan y enseñan estas tecnologías en cada uno de ellos, así como plantear una reflexión crítica sobre sus principales potencialidades y sus deficiencias más significativas.

Palabras clave: enseñanza/Grados/ Master/ Tecnologías de la Información Geográfica-TIG

\section{Abstract:}

This paper review current TIG-studies in Degrees and Masters' Spanish and European University. Using information from curricula available on websites, we've analyzed its main features, goals, duration, content, subjects, etc. The ultimate goal is to formulate an initial classification of these studies, know how to insert and teach these technologies in each of them, and raise a critical reflection on its main strengths and weaknesses.

Keywords: education/Degree/Master/Geographic Information Technologies-GIT

Bosque Sendra, J. (joaquin.bosque@uah.es); Gómez Delgado, M. (montserrat.gomez@ uah.es); Aguilera Benavente, F. (f.aguilera@uah.es); Rodríguez Espinosa, V.M. (victor. rodriguez@uah.es); Barreira González, P. (pablobarreiragonzalez@hotmail.com); Salado García, M.J. (mariaj.salado@uah.es. Unidad Docente de Geografía (Dpto. Geografía y Geología), Universidad de Alcalá 


\section{PROBLEMÁTICA DE LA ENSEÑANZA DE TECNOLOGÍAS DE LA INFORMACIÓN GEOGRÁFICA-TIG-}

La discusión sobre la enseñanza de los SIG-Sistemas de Información Geográfica y, más en general, de todas las TIG tiene cierta tradición en España; se han publicado varios trabajos sobre el tema y especialmente relevantes han sido las aportaciones que se hicieron en el V Coloquio de Geografía Cuantitativa del Grupo de Métodos Cuantitativos de la Asociación de Geógrafos Españoles-AGE (Zaragoza 1992; actas y comunicaciones disponibles en: http://age.ieg.csic.es/metodos/congresos/zaragoza92.html), entre ellas Bosque Sendra (1992), Bosque et al. (1992), Comas (1992). Con posterioridad, aunque en menor grado, es posible encontrar en la bibliografía algunas interesantes reflexiones al respecto, entre las que cabe destacar Chuvieco (1993), Bosque Sendra (1999) o Bosque Sendra (2000). Sin embargo, en ninguno de los coloquios y congresos que cada dos años organiza el mencionado grupo de la AGE (ahora Grupo TIG de la AGE) se ha vuelto a tratar esta problemática. Esto, en cierta media y cuanto menos, resulta curioso pues estos encuentros, consolidados ya como unas de las reuniones científicas más importantes sobre TIG en nuestro país y referente obligado en el ámbito de la investigación en esta materia, constituyen al mismo tiempo foros de discusión de todo lo relacionado con este tipo de tecnologías. Por ello, parece oportuno solicitar que se preste en estos Congresos alguna atención al tema y se reflexione sobre la enseñanza de estas tecnologías en diferentes niveles educativos.

Una posible causa de la escasa visibilidad de la vertiente "educativa" de las TIG en este tipo de encuentros sea tal vez que, a principios de los años noventa, se produjo una reforma importante de los planes de estudio universitarios en España, lo que condujo a una cierta discusión sobre cómo incluir estos temas en las nuevas enseñanzas. Pero ahora, cuando se está produciendo la aplicación del denominado Plan Bolonia, para la integración de la Universidad española en el Espacio Europeo de Enseñanza Superior-EEES, puede que sea oportuno retomar y/o profundizar en la discusión sobre este aspecto. En esta línea se pueden destacar algunos esfuerzos recientes de gran interés, como la encuesta de la Asociación Española de Teledetección-AET para determinar el estado de la docencia de esta tecnología en nuestro país (en http://www.aet.org.es/?q=encuesta-docencia-gral1) o el trabajo de Serra et al. (2012) sobre la situación de las TIG en la enseñanza superior y especializada en Cataluña. 
En la serie de trabajos antes mencionados ya se planteaban diversos problemas y cuestiones en relación a la enseñanza de las TIG en la Universidad española, entre las que, a modo de resumen, cabría destacar las referidas al tipo de profesional a formar (¿usuarios "inteligentes", administradores y desarrolladores de sistemas, consultores?) o la orientación/enfoque general que se debiera dar a los estudios de estas tecnologías (formación de usuarios para la aplicación de las TIG en tareas de ordenación del territorio, en sentido amplio, vs formación de creadores/generadores de datos geográficos digitales).

En esta misma línea, una cuestión importante que ahora es interesante plantear tiene que ver con el nivel de difusión que han tenido las TIG en los nuevos estudios de Grado que se han ido implantando en España y en Europa. ¿Cuántas asignaturas de estos temas se han incluido? 0 ¿qué nivel de profundidad tienen estos estudios?. Relacionado con lo anterior, ¿cuántos estudios de Máster incluyen enseñanzas sobre estos temas?; ¿existe coherencia entre los niveles de detalle y profundidad de las enseñanzas en estos dos niveles docentes?. Por último, también sería posible plantearse qué ocurre con las TIG en los estudios de doctorado.

A estas preguntas y a otras similares intenta responder este trabajo, realizando para ello una recopilación y revisión de un número importante de estudios TIG o que, al menos, incluyan asignaturas relacionadas con las TIG en sus planes de estudios.

Internet ha sido la principal fuente de información para realizar este análisis, consultando páginas electrónicas institucionales de los diferentes estudios (anexo 1). En la actualidad, es el medio más utilizado y eficaz para darse a conocer, para informar sobre características, requisitos, objetivos, etc. de los estudios y, en definitiva, en el marco competitivo en el que se mueven actualmente las Universidades española y europea, para captar potenciales estudiantes'. Por otra parte, en esta misma línea, la información recogida en estas páginas también es utilizada, cada vez más, en las evaluaciones de calidad a los que son sometidos los estudios por parte de agencias externas (por ejemplo, en España, la ANECA).

\footnotetext{
${ }^{1}$ La influencia del Plan Bolonia se deja también sentir en la información recogida en estas páginas electrónicas, más apreciable en las de estudios oficiales donde es más fácil encontrar cuestiones típicas de esta reforma de las enseñanzas: objetivos, listas de competencias a alcanzar, mecanismos de control de calidad, etc. En las de los otros estudios se mantiene un cierto enfoque clásico con descripción de asignaturas y contenidos, enumeración de profesorado, etc.
} 
Pero previamente es conveniente y oportuno definir las TIG, a qué técnicas nos referimos cuando hablamos de TIG. Bosque (1999) las define como "procedimientos desarrollados para reunir, manipular (analizar) la información geográfica, en especial aquella que está expresada en formato digital". Conforman un conjunto amplio de tecnologías, cada vez más difundidas, diseñadas y pensadas para gestionar, manejar y procesar información espacial.

Inicialmente se puede considerar bajo esa denominación diferentes tecnologías, temáticas y disciplinas académicas, entre ellas, sin intención de ser exhaus tivos: (a) Cartografía, una de las más antiguas, con orígenes que, seguramente, se remontan a la Antigüedad; (b) Geodesia, que hasta el Renacimiento europeo no se puede considerar como una materia técnica avanzada; (c) Fotointerpretación, surgida en el siglo XIX y, sobre todo, en el XX al amparo de la aparición y los avances en la fotografía, en general, y en la fotografía aérea, en particular, y sus amplias posibilidades en la obtención de datos sobre el territorio; (d) Fotogrametría, muy relacionada con la anterior, facilita las herramientas más potentes para poder obtener información espacial de imágenes aéreas; (e) Teledetección, similar a la Fotointerpretación pero utilizando una nueva fuente de imágenes, los sensores y receptores portados por satélites artificiales, surge ya avanzado el siglo XX; (f) SIG, surgen, casi simultáneamente a la Teledetección, como herramientas para el análisis de datos territoriales reunidos por muchas de las ya citadas técnicas; (g) Sistemas de Posicionamiento Global (GPS, GLONASS o futuro Galileo), se pueden considerar plenamente operativos en la década de 1990 como nuevo método para proporcionar la localización sobre el territorio, añadiendo nuevas posibilidades a la Geodesia y a la obtención de datos espaciales; (h) Infraestructuras de Datos Espaciales-IDE. iniciativas que surgen también hacia la década de 1990 como medio de difundir, con facilidad y eficacia, la enormidad de datos territoriales disponibles con el uso de toda esa panoplia de tecnologías, sistemas y servicios de búsqueda, acceso y difusión de información a través de Internet, así como de estándares y protocolos de armonización de la misma y de las tecnologías relacionadas. Estas iniciativas están adquiriendo gran importancia y difusión y son numerosas las IDE que, a todos los niveles (corporativo, local, regional, nacional, etc.) están en marcha; (i) Geografía Voluntaria o Participativa (VGI, acrónimo inglés), relacionada con la anterior y en la que personas e instituciones diversas, usando Internet, ponen a disposición de todos datos territoriales (buen ejemplo es Google Map o Google Earth, Goodchild, 2007); (j) Location-based services and mobile geospatial technologies, novísimas técnicas, en rápido crecimiento y de uso cada vez más masivo, orientadas a ofrecer 
servicios personalizados de diversa índole a usuarios, considerando la ubicación geográfica de los mismos y haciendo uso de tecnologías de comunicación en red, SIG, GPS, dispositivos telefónicos móviles o similares (Hurson y Gao, 2009).

En principio se podría pensar que los estudios relacionados con TIG deberían o podrían incluir enseñanzas de todas estas tecnologías, temáticas y disciplinas (fundamentos conceptuales, técnicos y aplicados) que, por otra parte, tienen muy diferente complicación y profundidad. Como veremos después, y como es fácil de prever, esto no es sencillo y no resulta viable en la mayor parte de las ocasiones. Lo habitual es que los diversos estudios (Grados, Máster, etc.) se vean obligados a realizar una selección entre las tecnologías y temáticas mencionadas en función de los diversos intereses que fundamentan su desarrollo. Esta situación puede dar origen a estudios TIG muy diferentes entre sí.

\section{LA BÚSQUEDA DE ESTUDIOS TIG EN LAS UNIVERSIDADES DE ESPAÑAY EUROPA}

Los estudios TIG en Universidades de España y Europa presentan una amplia variedad de posibilidades y de opciones. En España actualmente no existen Grados dedicados en exclusiva al estudio de TIG, aunque sí se pueden encontrar asignaturas relacionadas con ellas en varios Grados como, por ejemplo, en la mayoría de los Grados de Geografía. Algo similar se puede decir de los Grados de Ciencias Ambientales, que suelen incluir materias centradas en estos temas. En Universidades europeas, sin embargo, sí se ha podido constatar la existencia Grados dedicados en exclusiva, o casi, al estudio de TIG.

Por el contrario, es en el nivel de Máster donde la oferta de enseñanzas TIG es más amplia y diversa en nuestro país, como lo es también en el resto de Europa, encontrándose múltiples titulaciones que se podrían enmarcar en este ámbito.

En la tabla 1 se muestra la relación de estudios TIG (de Grado y Máster, en Universidades españolas y europeas) que se han analizado en este trabajo. No son todos los existentes; aunque no sea exhaustiva, se puede considerar que la selección realizada incluye un número amplio y bastante representativo de los diferentes tipos de enseñanzas españolas y europeas relacionadas con TIG. Es bastante posible obtener conclusiones claras en base a la información recogida en ese conjunto de enseñanzas. 
Tabla 1. Relación de estudios de Grado y Máster TIG en Universidades españolas y europeas

\begin{tabular}{|c|c|c|c|c|}
\hline NIVEL & DENOMINACION & AMEITO & \multicolumn{2}{|c|}{ UNIVERSIDADINSTITUCION } \\
\hline \multirow{2}{*}{$\begin{array}{l}\frac{8}{8} \\
0 \\
\frac{8}{0} \\
0\end{array}$} & Geomatica y Topogratia & \multirow{2}{*}{$\begin{array}{l}\frac{\Sigma}{c} \\
\frac{a}{\omega} \\
w\end{array}$} & $\begin{array}{l}\text { UEX-Extremadura } \\
\text { UJa- Jaen } \\
\text { ULPCC-Las Famas } \\
\text { ULe- Leön } \\
\text { UOv-Oriado }\end{array}$ & 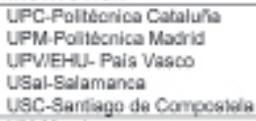 \\
\hline & Geografia & & $\begin{array}{l}\text { UAY-Autonoms Madnd } \\
\text { UAg-Rutbonoma Barcelons } \\
\text { UB-Larcelons }\end{array}$ & $\begin{array}{l}\text { UN-Murcia } \\
\text { UOw Ovodo } \\
\text { UPWE }\end{array}$ \\
\hline & & & $\begin{array}{l}\text { UCa- Camabria } \\
\text { UCM- Camplutense } \\
\text { UDQ- Gercona } \\
\text { ULe- León }\end{array}$ & $\begin{array}{l}\text { USal.Solamanca } \\
\text { USC-Santiago de Compoetola } \\
\text { US-Savila } \\
\text { UNa-Valladolid } \\
\text { UniZar-Zarageas }\end{array}$ \\
\hline & Ciencias Ambientales & & $\begin{array}{l}\text { UAl-Alcala de Henares } \\
\text { UB Barcelong } \\
\text { UGr-Granodo } \\
\text { UPVEEHU. Pais Vasco }\end{array}$ & \\
\hline & $\begin{array}{l}\text { Geographic Information } \\
\text { Technologins }\end{array}$ & EUROPA & $\begin{array}{l}\text { Newcable University (Rein } \\
\text { Pertamouh University (Reit }\end{array}$ & \\
\hline
\end{tabular}

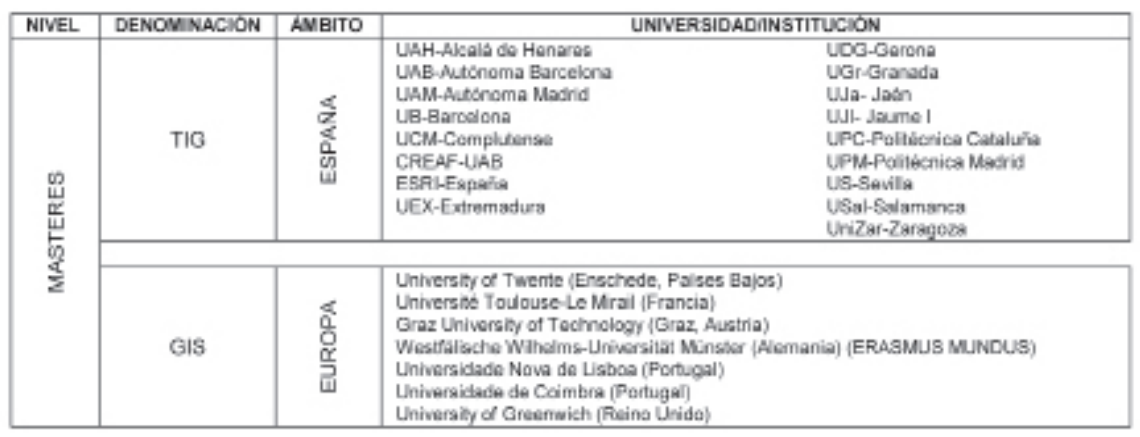

Fuente: Elaboración propia

Se ha seleccionado una muestra representativa de los Grados (en Geografía², Geomática y Topografía, Ciencias Ambientales) impartidos en Universidades españolas, así como algunos Grados y Másteres específicos TIG de Universidades europeas. En el caso de Másteres TIG españoles, sí se ha intentado recoger y analizar la totalidad de los impartidos hasta la fecha en nuestras Universidades.

Con objeto de facilitar la recopilación de información y su posterior análisis, se diseñó un sencillo formulario con el que se pretendía cubrir, en la medida en que la información incluida en las páginas electrónicas de las diferentes enseñanzas lo posibilitan, cuestiones que se consideraron relevantes y de interés para este estudio.

\footnotetext{
2En un reciente trabajo de Esparcia y Sánchez (2012) sobre los nuevos Grados en Geografía españoles se identifican 26 Universidades en las que se imparte este tipo de estudios.
} 


\begin{tabular}{|c|c|}
\hline NOMBRE DEL ESTUDIO & \\
\hline NIVEL & Grado/Postgrado \\
\hline TIPO DE ESTUDIO & OficialPropio \\
\hline UNIVERSIDAD & \\
\hline DIRECCION web & \\
\hline DURACION & Años/Créditos \\
\hline OEJETIVOS & ... a conseguir con la reslizsción de la enserianza \\
\hline COMPETENCIAS & .... que debefían obtenerge por los estudiantes al finalizer el estudio \\
\hline TIPOS GRALES. DE CONTENIDOS & Cartogretla, SIG, Teledeteccion, elc. \\
\hline ASIGNATURAS & ... más usuilese importantes, relacionadas con TIG \\
\hline
\end{tabular}

\section{RESULTADOS}

Organizado según tipo de estudio, en este apartado se presentan algunos de los resultados y conclusiones destacadas en relación a la presencia de TIG en la enseñanza universitaria española y europea.

\section{a) Estudios de Grado}

En España, la presencia de la enseñanza de TIG en estudios de Grado (con una duración de 4 años, 240 ECTS $^{3}$ ) se puede clasificar en dos grandes grupos: en el primero estarían los numerosos Grados de Geomática y Topografía, impartidos en Escuelas/Universidades politécnicas y, por lo general, vinculados a departamentos universitarios de Ingeniería Topográfica o similares.

En cierto modo estos Grados son herencia de los estudios de la antigua Ingeniería Técnica de Topografía y de la Ingeniería Superior en Geodesia y Cartografía. Incluyen en sus planes de estudio una buena cantidad de asignaturas TIG (SIG, Teledetección, Cartografía, Fotogrametría y Geodesia, principalmente) y se puede considerar que son los estudios que más espacio otorgan a estas tecnologías en la enseñanza universitaria española; entre $30-50 \%$ de los créditos se dedican a su estudio. Junto a estas materias se añaden otras que pueden considerarse básicas, como las Matemáticas y algunas partes de la Física sobre las que, en

\footnotetext{
${ }^{3}$ Los ECTS (European Credit Transfer System) representan entre 25-30 horas de aprendizaje y valoran el tiempo invertido por el alumno (asistencia al aula, estudio, trabajo fuera del aula, etc.) en adquirir las competencias del programa de estudio.
} 
parte, se sustentan la Geodesia, la Cartografía, etc.

En conjunto se trata de una enseñanza de marcado carácter ingenieril y aplicado y cuyo objetivo explícito es capacitar a los estudiantes para la realización de proyectos relacionados con la medida, explotación y otras varias acciones sobre el territorio, empleando para ello las TIG: "planificar, proyectar, dirigir, ejecutar y gestionar procesos de medida, sistemas de información, explotación de imágenes, posicionamiento y navegación (...) modelización, representación y visualización de la información territorial en, bajo y sobre la superficie terrestre".

En estos Grados aparece un alto contenido en competencias ${ }^{4}$ relacionadas con TIG, siendo los estudios que más se asemejan a los existentes en otras Universidades europeas. Cabe destacar algunas, como: (1) "conocimiento, aplicación $y$ análisis de los procesos de tratamiento de imágenes digitales e información espacial, procedentes de sensores aerotransportados y satélites" o (2) "diseño, producción y difusión de la cartografía básica y temática"; "implementación, gestión y explotación de SIG".

Por otra parte, estarían los Grados de carácter territorial/geográfico, principalmente los de Geografía y Ordenación del Territorio y, en menor medida, los de Ciencias Ambientales. Incluyen algunas materias relacionadas con TIG, no demasiadas. En ellos las TIG tienen una componente instrumental auxiliar aunque muy significativa, pues resultan fundamentales en la adquisición de competencias de carácter complejo, como las relacionadas con la Ordenación del Territorio o la Evaluación de Impacto Ambiental.

En Grados de Geografía es posible advertir diferencias notables con los de Geomática en cuanto a objetivos; mucho más prácticos y centrados en proyectos en el caso de estos últimos, los objetivos en los de Geografía son del tipo: (1) "manejar técnicas específicas de análisis e interpretación del espacio geográfico: fuentes de información, trabajo de campo, fotointerpretación, teledetección, tratamiento de datos y estadística, cartografía y SIG" o (2) "aplicación de los métodos de trabajo y de las TIG para abordar los estudios territoriales, medioambientales y paisajísticos

\footnotetext{
"El concepto de competencia, en el contexto del EEES, se define como "combinación dinámica de atributos, en relación a conocimientos, habilidades, actitudes y responsabilidades, que describen los resultados del aprendizaje de un programa educativo o lo que los alumnos son capaces de demostrar al final del proceso educativo" (Montero, 2010).
} 
a diferentes escalas de análisis".

Las competencias enumeradas parecen, en algunos casos, más concretas: "utilizar la información geográfica como instrumento para la interpretación del territo- rio"; "expresar información cartográficamente"; "conocer las capacidades de Ios SIG en la gestión y planificación de proyectos"; "elaborar cartografías temáticas e inventarios del medio físico para la evaluación, planificación y ordenación del territorio"; "conocer la naturaleza de la información geográfica en formato digital: geometría, topología y atributos temáticos".

Las TIG presentes en sus planes de estudios son, esencialmente, la Cartografía (muy frecuente en muchos Grados), SIG y, en menor medida, Teledetección y Fotointerpretación; son habituales asignaturas con denominaciones clásicas como Cartografía básica, Cartografía temática, Introducción a las TIG, SIG raster, SIG vectorial, etc. En general se puede considerar que en torno a un 10-15\% de la carga docente de este tipo de estudios se dedica a temas TIG, un cierto incremento respecto a lo que ocurría en las anteriores Licenciaturas en Geografía (Burriel, 2004). Pero no se termina de aclarar el nivel de profundidad con que se tratan los temas TIG en estos Grados y es de temer que, dado el número de créditos dedicados a estas técnicas, no se puedan obtener completamente las competencias pretendidas (figura 1).

Figura 1. Grados en Geografia (seleccionados) en Universidades españolas. ECTS dedicados a asignaturas especificas TIG sobre total del estudio.

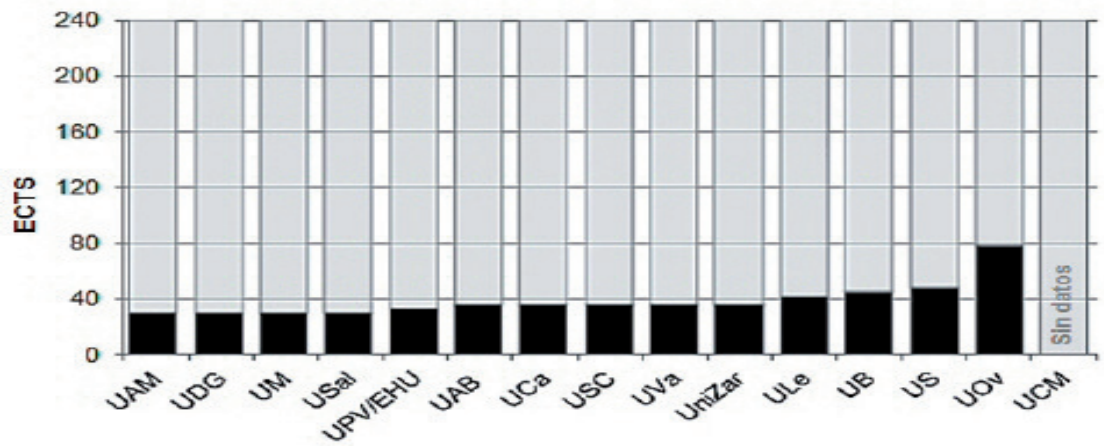

Fuente: Elaboración propia

En los Grados de Ciencias Ambientales, por su parte, la presencia de TIG es más reducida, siendo los SIG y, en menor medida, la Cartografía y la Teledetec- 
ción las más habituales. El número de asignaturas relacionadas que se incluyen en sus planes de estudio se limita a una o dos, tres como mucho; una carga docente que no suele superar los 10-12 ECTS y que tan sólo representa en torno a un 5\% del total de carga del estudio.

El objetivo de muchos de estos Grados se suele enunciar como “...formación adecuada para abordar los problemas ambientales desde diversos ámbitos del conocimiento, desde conocimientos teóricos y prácticos de las ciencias naturales y sociales hasta las tecnologías necesarias para aplicar estos conocimientos en la práctica...", o de esta otra manera "...crear expertos en el conocimiento de las relaciones entre las actividades humanas y el medio físico y biológico, en sus implicaciones socioeconómicas, y en las aplicaciones tecnológicas para la prevención y corrección de los problemas ambientales...". Y para conseguirlo se establecen competencias que, en lo referido a TIG, resultan un tanto vagas y generales (como “... manejo y aplicación de SIG e interpretación de imágenes de teledetección para aplicaciones ambientales...") y que, de nuevo, no es seguro que sea posible su consecución a la luz de la dedicación de créditos prevista en los planes de estudio.

En resumen, a nivel de Grado las TIG se enseñan en la Universidad española en tres tipos de estudios. Por una parte, un tipo más ingenieril y con mayor peso de las técnicas centradas en la obtención de los datos geográficos y, por otra, los Grados más relacionados con las Ciencias Sociales y los estudios que podríamos denominar "de letras".

El tercer ámbito donde se pueden estudiar, en cierto volumen, las TIG es precisamente este nuevo estudio de Ciencias Ambientales. Surgido en los últimos veinte años con bastante éxito, es un título "de ciencias" que compite tanto con otras carreras "de ciencias" (Biología, Químicas, etc.) como con alguna "de letras", como la renovada Geografía (Puyol et al., 2012).

En relación a la presencia de las TIG en estos estudios, es posible establecer una especie de gradación decreciente en cuanto a créditos dedicados a este tipo de tecnologías, desde los Grados en Geomática (donde, como hemos visto, la presencia de las TIG es significativa) hasta los de Ciencias Ambientales (con una presencia casi testimonial).

En varias Universidades europeas existen Grados dedicados exclusiva- 
mente a TIG. Aquí se han analizado, a modo de ejemplo, dos de los que se imparten en Universidades del Reino Unido (las de Newcastle y Portsmouth). La Universidad británica presenta ciertas peculiaridades en la adopción y adaptación al EEES, pero se ha considerado que los casos seleccionados pueden servir para conocer las características de estos estudios a nivel europeo y comprobar si existen diferencias significativas con los que se imparten en España.

En estos Grados británicos los objetivos resultan un tanto generales y no se hacen demasiadas referencias a TIG: "... to develop and refine student's intellectual and critical abilities so that they can define, investigate, analyse and synthesise problems, form judgements, make decisions and demonstrate their competence in such skills... ". Sin embargo, la formulación de competencias sí es precisa y clara, entre ellas: (1) "... combine and interpret different types of geographical evidence..."; (2) "... apply a range of techniques for the analysis of geographical data and interpret the outcomes..."; (3) "... effectively present geographical information..."; (4) "... perform applications programming appropriate to GIS...".

En general, en estos Grados se lleva a cabo una ampliación de los temas TIG estudiados; se añaden cuestiones de fundamentos (por ejemplo, Matemáticas y Bases de Datos) y, además, la presencia de TIG es destacada, añadiéndose a las habituales (Cartografía, SIG, Teledetección) otras menos habituales en Grados similares como la Fotogrametría y la Geodesia. Además se dedica una cierta cantidad de la actividad docente al estudio de algoritmos espaciales y a la programación enfocada a problemas geográficos.

Tienen, por lo tanto, cierta semejanza con los actuales Grados de Geomática y Topografía que se realizan en España, pero sin la fuerte especialización de estos en la Topografía y en temas similares.

\section{b) Estudios de Máster}

En España se han analizado dieciséis estudios de Máster relacionados de manera importante con TIG, algunos de ellos de tradición superior a los diez años. Más de la mitad son títulos propios de cada Universidad (o de empresas privadas) y el resto (unos 6) son estudios oficiales reconocidos por el Estado español (figura 2). 
Figura 2. Másteres TIG en España (2012), según tipo de estudio

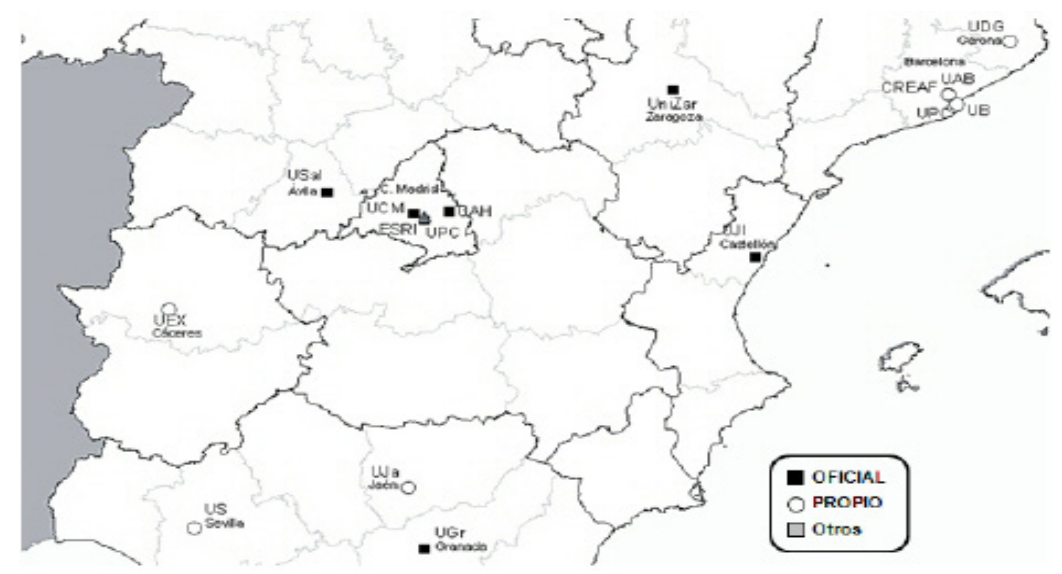

Fuente: Elaboración propia

En su mayoría se ajustan a la modalidad presencial o semipresencial (en Universidad de Salamanca, Extremadura y Politécnica de Cataluña) y sólo dos son estudios totalmente virtuales (Universidad de Jaén y Gerona). En ellos se suele imponer limitación de matrícula (entre 20 y 35 alumnos es el límite fijado en la mayor parte de los de tipo presencial, ampliándose hasta 60 u 80 en los virtuales, figura 3) y, habitualmente, se imparten durante un año académico, si bien hay ejemplos, como en la Universitat Autònoma de Barcelona o en la Universitat Jaume I, donde el estudio se supera este período.

Figura 3. Límite de matrícula ( $n^{0}$ alumnos admitidos) en Másteres TIG en España (2012)

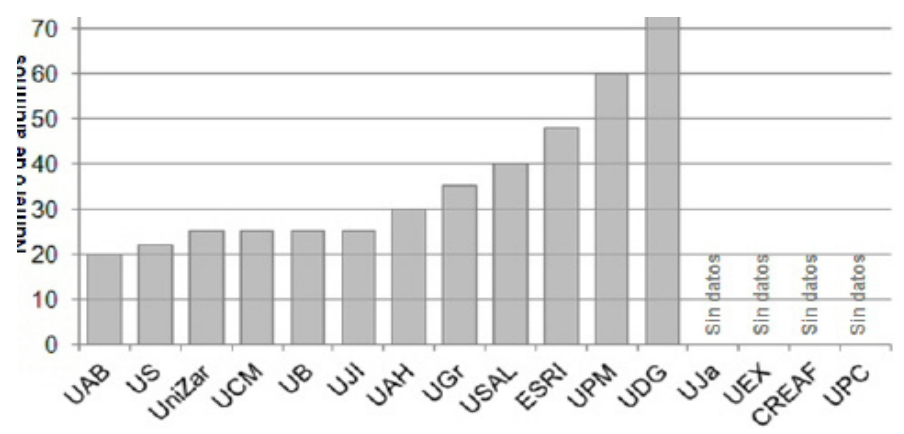

Fuente: Elaboración propia 
En conjunto, casi tres cuartas partes de estos estudios se realizan y/o dirigen por departamentos de Geografía. El resto de Másteres están encabezados por departamentos de Ingeniería Cartográfica de Universidades o Escuelas Politécnicas (los mismos que, en gran medida, encabezan la enseñanza de los Grados de Geomática y Topografía), aspecto que quizá esté incidiendo significativamente en el enfoque del estudio, más volcado a la formación de personal dedicado a la captura y creación de datos geográficos.

Diferente situación es la que se puede detectar en aquellos Másteres que parten desde departamentos de Geografía, más orientados a la preparación de usuarios (más o menos capacitados, "inteligentes") de las TIG, especialmente de los SIG.

La observación de los objetivos que se plantean en estos estudios parece confirmar estas primeras apreciaciones. Existen diversas versiones de objetivos dependiendo del enfoque general del Máster: (1) “... dominar los conceptos y herramientas básicas de las TIG..."; (2) "... formar profesionales para el trabajo con datos espaciales en empresas e instituciones..."; (3) "... aportaciones y aplicación de las TIG a la ordenación del territorio, análisis territorial u ámbitos específicos...".

Para conseguirlos se suelen enumerar algunas competencias comunes a todos los estudios analizados, entre ellas: "... ser capaz de realizar operaciones de gestión, análisis y presentación de la información geográfica en el entorno de los SIG..."; "... ser capaz de comprender, manejar, interpretar y analizar imágenes de satélite y fotografías aéreas..."; "... ser capaz de pensar (concebir), elaborar, utilizar e interpretar mapas sencillos...". Una formulación de competencias que, como se puede advertir, no se diferencia excesivamente de la que se puede encontrar en estudios de Grado, mostrando de nuevo la indefinición que puede existir en este aspecto entre los dos niveles de la enseñanza universitaria española.

Aunque los contenidos más usuales varían bastante entre Másteres, en general se puede decir que los relativos a SIG son incluidos en todos ellos. También la Cartografía está ampliamente representada en los contenidos de estos estudios, si bien con diferencias en cuanto a su importancia y peso en el temario de cada uno de ellos. Por su parte, la presencia de la Teledetección sólo se puede considerar relevante en la mitad de los Másteres analizados y la de otras técnicas (Geodesia, Fo- 
togrametría, GPS, etc.) es minoritaria. Estos contenidos, como se puede suponer, son más habituales en Másteres más "técnicos" (los que surgen de las Escuelas de Ingeniería), en los que también es habitual encontrar contenidos relacionados con las IDE.

De esta forma, las asignaturas concretas que se imparten también varían ampliamente entre Másteres (en cuanto a nombre y planteamiento), pero quizá se pueda considerar que existe una cierta coincidencia en la aparición de algunas materias, con contenidos similares: Fundamentos Cartografía (Definición. Sintaxis cartográfica. Lectura de mapas); Fundamentos SIG (Definición. Modelos de datos. Métodos de análisis. Aplicaciones SIG); Fundamentos Teledetección (Sensores. Imágenes. Tratamiento visual y digital); Análisis espacial o geográfico (Algebra de mapas. Transformación de datos espaciales. Estadística espacial).

En ninguno de los Másteres revisados se ha podido constatar la presencia (en contenidos y/o asignaturas concretas) de las recientes tecnologías mencionadas en la introducción del trabajo. Hay que tener en cuenta que, evidentemente, existe siempre un desfase temporal entre la aparición y desarrollo de cualquier nueva técnica, tecnología o metodología y su estudio formal en un título reglado. De ese modo, temas relacionados con location-based services and mobile geospatial technologies no se han encontrado representados con claridad.

Por lo que se refiere a Másteres similares impartidos en Universidades europeas, en este trabajo se han analizado los de siete Universidades (tabla 1). En principio, se advierte que se trata de estudios relativamente más avanzados que los que se desarrollan en España. Están dirigidos, sobre todo, a la preparación de usuarios TIG para que puedan aplicar estas tecnologías a la resolución de problemas concretos en empresas e instituciones diversas.

Entre las TIG, no sólo incluyen en sus planes de estudio las habituales SIG - Cartografía, también se puede comprobar la presencia de técnicas más dirigidas a la obtención de datos e información, como Fotogrametría o Teledetección (si bien no queda claro el nivel de profundidad que se alcanza en estas cuestiones), o, en varios casos, la de algunos nuevos desarrollos tecnológicos. En ellos también tiene presencia destacada la programación informática, que aparece como materia en la mayoría de los Másteres analizados. En este sentido, y en otros, presentan ciertas similitudes con los Másteres que se imparte en las Universidades catalanas, estu- 
diados recientemente en el trabajo de Serra et al. (2012).

Para terminar, ¿qué se puede decir de los estudios de Doctorado en su relación con TIG?. En España se ha producido recientemente una nueva revisión de las normas administrativas sobre este tipo de estudios. Ahora el estudiante de Doctorado no está obligado a recibir actividades docentes de importancia; se tiene que dedicar, casi exclusivamente, a elaborar su trabajo de investigación y a redactar una tesis, pues cuenta con un plazo de tiempo limitado para ello. Buscando en Universidades españolas parece que sólo en la de Alcalá (UAH) existe un Doctorado en el que las TIG tienen un protagonista (http://geogra.uah.es/ joomla/index.php/docencia/ct-menu-item-35). En el resto no existen estudios iguales o similares; puede encontrarse algunos Doctorados con otras temáticas en los que las TIG tienen algún papel auxiliar, pero no son centrales como en la UAH. Tampoco en Europa es fácil encontrar estudios con este planteamiento, ni tan siquiera en el prestigioso ITC holandés (University of Twente).

Para calibrar el interés y utilidad de este tipo de planteamiento del Doctorado se pueden consultar y analizar los resultados, tesis doctorales defendidas, etc.; en la dirección anterior, por ejemplo, también se enumeran las tesis presentadas y defendidas en los más de veinte años de existencia del Doctorado de la UAH. En cualquier caso, no es fácil concluir algo significativo con un solo caso de estudio, pero creemos que se proporciona alguna información útil sobre este tema.

\section{DISCUSIÓN Y CONCLUSIONES}

Las TIG vienen experimentando desde hace algunas décadas una evolución espectacular, una rápida difusión e implantación cada vez más evidente en cualquier ámbito profesional. El carácter "expansivo" de estas tecnologías también se ha dejado notar en el ámbito académico; la enseñanza de TIG despierta cada vez mayor interés y crece la preocupación por formar profesionales que utilicen y apliquen este tipo de tecnologías.

Se asiste, así, a la frecuente aparición de gran cantidad de estudios TIG, generales o más especializados. También se ha incrementado la oferta de estos estudios en los diferentes niveles de la educación universitaria, tanto en España como en el resto de Europa. 
En este trabajo se ha llevado a cabo una primera aproximación a este tipo de estudios para conocer sus principales características y saber cómo se aborda en ellos la enseñanza de TIG. Algunas conclusiones de cierto interés se han ido apuntando a lo largo del texto y aquí se señalan de nuevo y se incide en otras que se consideran igualmente interesantes.

En la Universidad española, a diferencia de lo que sucede en otros países europeos, no existen estudios de Grado dedicados en exclusiva a TIG. Su enseñanza suele formar parte de estudios más generalistas, como los Grados de Geografía/ Ordenación del Territorio o de Ciencias Ambientales. En ellos la presencia de TIG, en cuanto al número de asignaturas y créditos dedicados en sus planes de estudio, no suele ser muy importante; se podría decirque, en algunos casos, es casi testimonial.

Sin embargo, en otros Grados, como los de Geomática y Topografía, las TIG aparecen de forma significativa en sus planes de estudio. En este sentido presentan similitud con los Grados TIG europeos que se han analizado en este trabajo, con una amplia representación de asignaturas sobre TIG y centrados en la formación más aplicada de las mismas.

De esta forma, cabría plantear algunas reflexiones sobre la conveniencia de implantar en el Grado español titulaciones específicas de TIG. Preguntarse sobre el motivo por el que estos estudios no existen en este nivel en la Universidad española. Y preguntarse, de igual forma, si la formación que en estas materias se da en los Grados analizados cubre suficientemente la formación básica del alumnado y lo prepara para enfrentarse, más adelante, al nivel más avanzado, especializado, que representan los Másteres.

En muchos de los Másteres revisados es frecuente encontrar asignaturas sobre nociones básicas o fundamentos de, al menos, las TIG clásicas. ¿Hasta qué punto resulta necesario o conveniente incidir en éstas cuestiones en un estudio avanzado?.

La oferta de Másteres relacionados con TIG en las Universidades españolas, tanto de carácter oficial como estudios propios universitarios, es amplia. En muchos casos, se ve complementada con una oferta también amplia y frecuente de estudios especializados y específicos, impartidos desde el ámbito académico y/o desde la empresa privada. 
Como en el caso de los Grados, en los Másteres TIG españoles se pueden advertir varias orientaciones, relacionadas con la forma de enseñar estas tecnologías y el tipo de formación que se persigue en cada uno de ellos. Dependiendo, en cierta medida, del origen de la iniciativa, es posible encontrar, por un lado, Másteres más volcados a la formación de personal dedicado a la captura y creación de datos geográficos (estudios relacionados con Universidades y/o Escuelas Politécnicas) y, por otro, estudios en los que hay mayor preocupación por preparar y formar usuarios "inteligentes" de TIG (relacionados con departamentos de Geografía o similares).

Las diferencias con los Másteres europeos (los siete analizados) no son muy significativas. Tal vez lo más destacado es constatar que, en general, son enseñanzas un tanto más avanzadas, en el sentido de que presentan una clara orientación hacia la aplicación de TIG en la resolución de problemas concretos de empresas e instituciones/organismos diversos, así como la mayor presencia de la programación informática en sus planes de estudio (por ejemplo, en su aplicación a SIG, para extender y/o personalizar funcionalidades de las herramientas, para su implementación en entornos web, etc.).

Al igual que en los Másteres españoles, en estos estudios no se aprecia una presencia clara y significativa de materias y/o contenidos relacionados con las TIG de reciente aparición y desarrollo que, por otra parte, suelen ser ofertados como cursos de extensión universitaria o de empresas privadas relacionadas con las TIG. Tampoco parecen resueltos aquí algunos aspectos relativos a la formulación de competencias.

Otra de las reflexiones destacadas de este trabajo tiene que ver con la falta de coordinación que, en este sentido, se detecta entre los dos niveles de la enseñanza universitaria (Grados y Másteres). La formulación de competencias de ambos no se diferencia excesivamente y, lo que puede ser más preocupante, no parece existir continuidad en las mismas; si los planes de estudio estuviesen bien elaborados debería existir una jerarquización de competencias entre ambos niveles, y eso no es tan claro en los estudios aquí analizados.

Una cuestión igualmente problemática en este sentido, que también se ha evidenciado en este trabajo, tiene que ver con el hecho de que, en no pocas oca- 
siones, la formulación de las diferentes competencias suele resultar poco realista en cuanto al tiempo que se prevé para su consecución. Por ejemplo, refiriéndonos a SIG, en varios de los Másteres revisados tan sólo se prevé para su estudio seis ECTS; ¿es posible alcanzar así la totalidad de competencias planteadas?. Parece difícil. Este es un problema bastante común que aparece en las evaluaciones que, por ejemplo, realiza en España la ANECA sobre los diferentes estudios universitarios; se plantean competencias pero no se incluye tiempo suficiente para su obtención.

Resulta necesario que los planes de estudio estén bien formulados, que sean más explícitos en las competencias de las diferentes materias que incorporan, incluyendo con más frecuencia términos como "básico" o "nivel básico", en el caso de los Grados, para diferenciarlas expresamente del nivel avanzado que representan los estudios de Máster.

\section{BIBLIOGRAFÍA}

- Alegre Nadal, P. (1992): "La docencia de la Teledetección", en V Coloquio de Geografía cuantitativa. Actas. Comunicaciones (Zaragoza, septiembre de 1992). Servicio de Publicaciones de la Universidad de Zaragoza, 1992. Disponible en: http://age.ieg.csic.es/metodos/zaragoza92/1992_19_alegre.pdf.

- Bosque Sendra, J. (2000): "Nuevas perspectivas en la enseñanza de las tecnologías de la información geográfica", en Serie Geográfica, nº 8, pp. 25-34. Disponible en: http://dialnet.unirioja.es/servlet/articulo?codigo=190809.

- Bosque Sendra, J. (1999): "La Ciencia de la Información Geográfica y la Geografía”, en VII Encuentro de Geógrafos de América latina. Publicaciones CD, Inc. (CD-ROM), San Juan de Puerto Rico, 15 págs.

- Bosque Sendra, J. (1992): "La enseñanza de los Sistemas de Información Geográfica", V Coloquio de Geografía cuantitativa. Actas. Comunicaciones (Zaragoza, septiembre de 1992). Servicio de Publicaciones de la Universidad de Zaragoza, 1992. pp. 47-58. Disponible en: http://age.ieg.csic.es/metodos/zaragoza92/1992_03_bosque.pdf.

- Bosque Sendra, J.; Chuvieco Salinero, E.; Domínguez, L. y González, R. (1992):"Aproximación a la problemática de la Didáctica de los SIG", en Comunicaciones $1^{\circ}$ Congreso AESIGYT, Los Sistemas de Información Geográfica en la gestión territorial (Madrid, abril de 1992), AESIGYT, pp. 126-138.

- Burriel de Orueta, E.L. (2004): "La base formativa común en la enseñanza universitaria de la Geografía en España", en Boletín de la Asociación de Geógra- 
fos Españoles, $n^{0}$ 38-2004, pp. 47-70. Disponible en: http://dialnet.unirioja.es/ servlet/articulo?codigo $=1079115$.

- Comas, D. y Donaire Beito, J.A. (1992): "La enseñanza de los SIG. Reflexiones didácticas", en V Coloquio de Geografía cuantitativa. Actas. Comunicaciones (Zaragoza, septiembre de 1992). Servicio de Publicaciones de la Universidad de Zaragoza, 1992, pp. 75-89. Disponible en: http://age.ieg.csic.es/metodos/ zaragoza92/1992_05_comas\&donaire.pdf.

- Chuvieco, E. (editor) (1993): "La enseñanza de la Teledetección en España", en Serie geográfica, $n^{0}$ 2.Esparcia Pérez, J. y Sánchez Aguilera, D. (2012): "De la teoría a la práctica. El proceso de diseño e implantación de los Grados de Geografía en las Universidades españolas", en Boletín de la Asociación de Geógrafos Españoles, n 58-2012, pp. 405-427. Disponible en: http://dialnet. unirioja.es/servlet/articulo?codigo $=3886904$.

- Goodchild, M. (2007): "Citizens as sensors: the world of volunteered geography", en GeoJournal 69 (4), pp. 211-221. Disponible en: http://link.springer.com/ article/10.1007\%2Fs10708-007-9111-y.

- Hurson, A.R. y Gao, X. (2009): "Location-Based Services", en Encyclopedia of Information Science and Technology. Second Edition, IGI Global, pp. 24562461. Disponible en: http://www.igi-global.com/chapter/location-based-services/13929.

- Montero Curiel, M. (2010): "El Proceso de Bolonia y las nuevas competencias", en Tejuelo, $n^{\circ} 9$ (2010), pp. 19-37. Disponible en: http://iesgtballester.juntaextremadura.net/web/profesores/tejuelo/vinculos/articulos/r09/03.pdf

- Pujol, H.; Garcia Ramón, M.D. y Ortiz, A. (2012): "El profesorado universitario de Geografía en España y sus trayectorias profesionales: una mirada de género", en Boletín de la Asociación de Geógrafos Españoles, $n^{0}$ 59-2012, pp. 323-344. Disponible en: http://ddd.uab.cat/pub/artpub/2012/4946/bolage_ a2012n59p323.pdf.

- Santos Preciado, J.M. (1990): "La enseñanza en la Universidad española de los métodos cuantitativos en Geografía", en IV Coloquio de Geografía cuantitativa. Actas. Comunicaciones (Mallorca, septiembre de 1990). Servicio de Publicaciones de la Universtitat de les Illes Balears, 1990, pp. 261-296. Disponible en: http://age.ieg.csic.es/metodos/1990_Palma/1990_16_Santos.pdf.

- Serra, P.; Pou, M. y Pons, X. (2012): "La docència en Ciència i Tecnologia de la Informació Geogràfica a Catalunya (2006-2009): una aproximació quantitativa", en Documents d'Anàlisi Geogràfica 2012, vol. 58/3, pp. 497-517. Disponible en: http://www.raco.cat/index.php/DocumentsAnalisi/article/view/259387. 


\section{Anexo 1- Direcciones de Internet-}

\begin{tabular}{|c|c|c|}
\hline Univergidad & Denominacion & Dirección wab \\
\hline UAH & TIB & 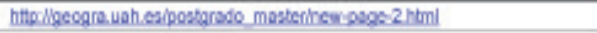 \\
\hline UCM & TIG & 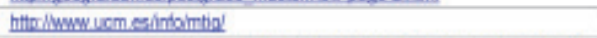 \\
\hline UPM & Inganieria Goodsaica y Cartografia & 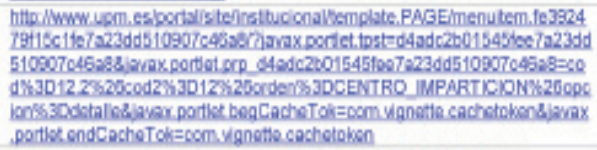 \\
\hline ESRI & SIG deESRI & 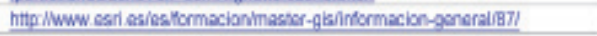 \\
\hline Unizar & $\begin{array}{c}\text { TIC para la Ordanación del Theribro SIC } \\
\text { y Telesetection }\end{array}$ & 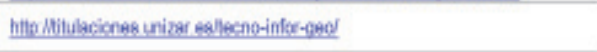 \\
\hline UGir & $\begin{array}{l}\text { Analisisis Googriffco on la Gestón del } \\
\text { Terriborio. TIG }\end{array}$ & the-Wmasteres ugr.es/masteragot \\
\hline UNa & $\begin{array}{c}\text { Evaluacon y Gestion de la Calidad de is } \\
\text { hf Geogrifica }\end{array}$ & 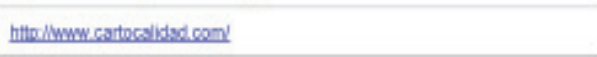 \\
\hline us & $\begin{array}{l}\text { 810: Planifcescián, Ondsonaciòn Taritarial } \\
\text { y Modio Ambiente }\end{array}$ & 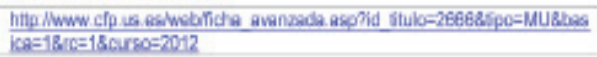 \\
\hline UEX & TIQ 819 y Tulatulactión & 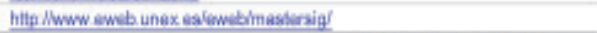 \\
\hline UA & Froducsia Carbog Afica i 810 & 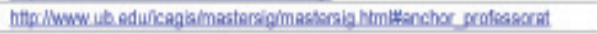 \\
\hline CREAF & Teiedeleccoo i \$IG & 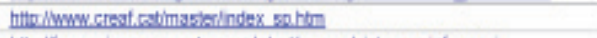 \\
\hline UPC & SKG & 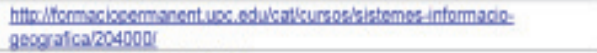 \\
\hline UAB & TIS & 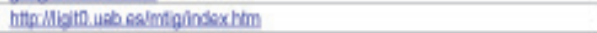 \\
\hline UNIGIS & Gestion de SIG & 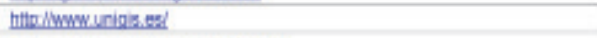 \\
\hline USAL & $\begin{array}{l}\text { Gootocnoiogias Cartogtticas on } \\
\text { hogenieria Y Naultectura }\end{array}$ & 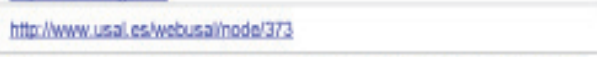 \\
\hline WI & $\begin{array}{l}\text { Tecnebois Geoespacial/Gecopatad } \\
\text { Tecthnologies }\end{array}$ & 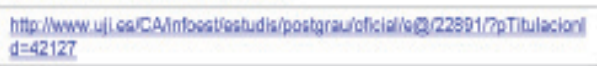 \\
\hline Greenwich & $\begin{array}{l}\text { Geographical hformation syatoma wifh } \\
\text { Ramase Sersing }\end{array}$ & 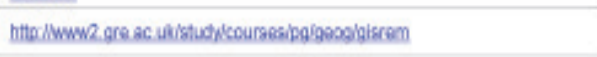 \\
\hline Twente & $\begin{array}{l}\text { Geo-Iformation Scence and Esth } \\
\text { Oboervation tor GEOINFORMATKCS }\end{array}$ & 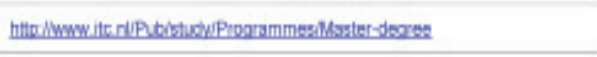 \\
\hline $\begin{array}{l}\text { Toulouse-Le } \\
\text { Mrail }\end{array}$ & Master 2 GEOMATIQUE SIGMA & 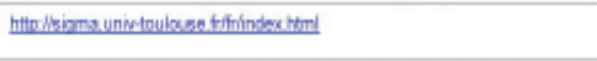 \\
\hline $\begin{array}{l}\text { Graz Un. of } \\
\text { Technology }\end{array}$ & Geo Spatis Tecthoiogies & 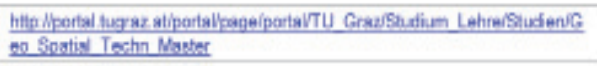 \\
\hline $\begin{array}{l}\text { Westfalsche } \\
\text { Wiheirs.- } \\
\text { Universitat } \\
\text { Mungter }\end{array}$ & Scrence in Geospotial Technologes & Hillo limastergestachinfol \\
\hline Coimbra & TIO & 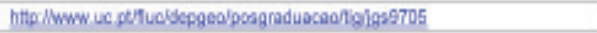 \\
\hline Nova de Lisbon & Cièncis e SIG & 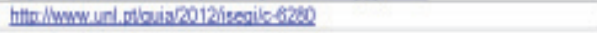 \\
\hline
\end{tabular}

\begin{tabular}{|c|c|c|}
\hline Universidad & Denominación & Dirección web \\
\hline UAB & Goografia y Orden. Terrbono & 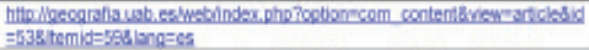 \\
\hline UAM & Ceografia y Orden. Temtorio & http-lWww uam. ealdepartamentosflioyletras/gecgrefiadbeencia htral \\
\hline UB & Googrstis & 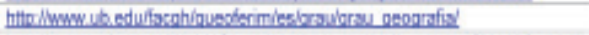 \\
\hline $\mathrm{UCa}$ & Goografia y Ordon. Terrtorio & 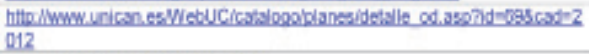 \\
\hline UCM & Ceografia y Orden. Temborio & http /WWw cacm esintorgeografia \\
\hline UoG & Goografia Ord. Temt y Gest. Anb. & 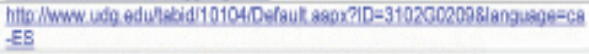 \\
\hline ULe & Geografia y Orden. Terrtorio & 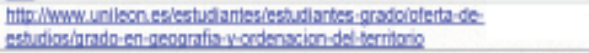 \\
\hline UM & Geografia y Orden. Territorie & 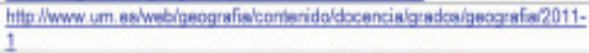 \\
\hline UON & Geografia y Orden. Territorio & 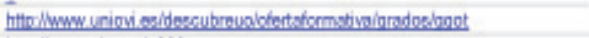 \\
\hline UPVIEHU & Geografia y Orden. Territonio & 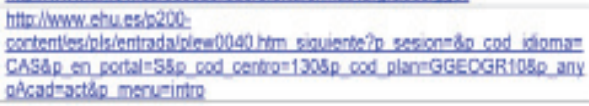 \\
\hline
\end{tabular}




\begin{tabular}{|c|c|c|}
\hline USal & Googratio & 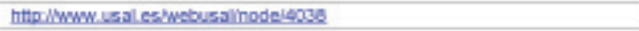 \\
\hline usc & Gecografia y Orden. Terriborio & 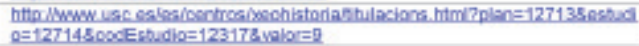 \\
\hline us & Geografla y Gestion Terre & http-MWww us es/eshulios/grados/plan $1647 p=4$ \\
\hline UVa & Geogrefie y Orden. Territorio & 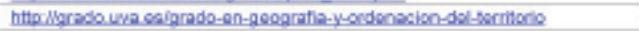 \\
\hline UnZar & Gsografia y Orden. Terribono & 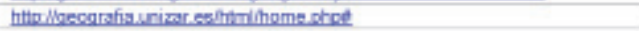 \\
\hline
\end{tabular}

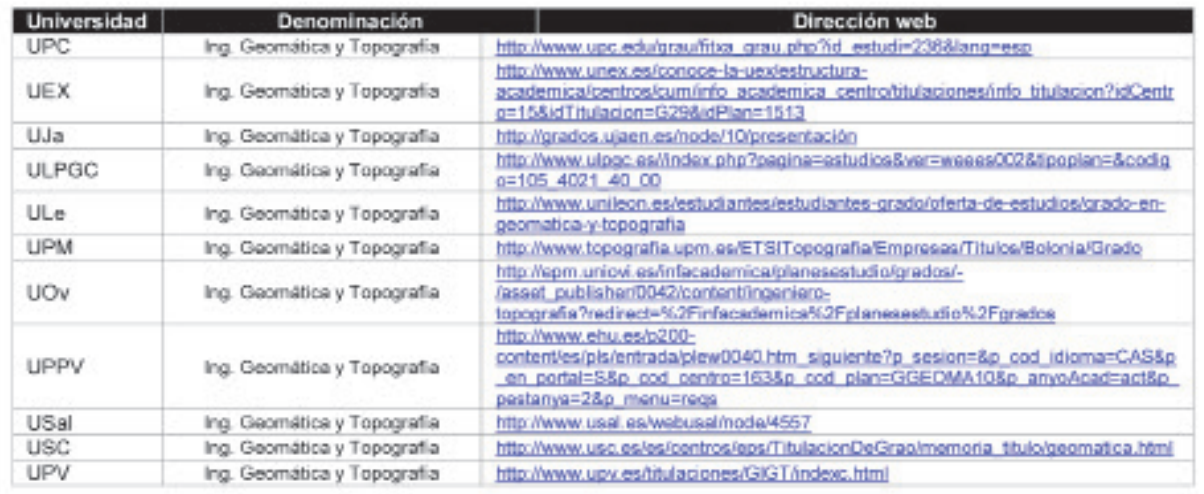

\begin{tabular}{|c|c|c|}
\hline Universidad & Denaminación & Dirección web \\
\hline UB & Concias Ambientales & 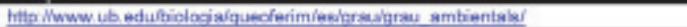 \\
\hline UAH & Cinncias Ambientales & 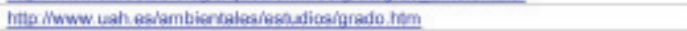 \\
\hline UPVIEHU & Cencias Ambientsles & 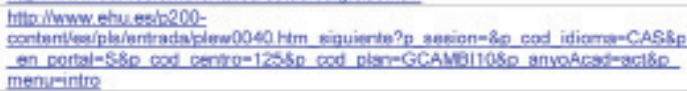 \\
\hline UGr & Cencias Ambientales & 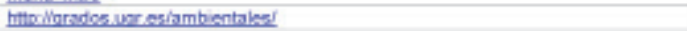 \\
\hline
\end{tabular}

